\title{
Editorial Message: Special Issue on Advances in Parallel and Distributed Computing for Fuzzy Systems
}

\author{
Guoqing Xiao' ${ }^{1}$ Kenli $\mathrm{Li}^{2} \cdot$ Maozhen $\mathrm{Li}^{3} \cdot$ Lipo Wang ${ }^{4}$
}

Published online: 17 August 2019

(C) Taiwan Fuzzy Systems Association 2019

Artificial Intelligence (AI) has grown substantially in popularity in recent years. Much research has been done in both academia and industry, with numerous applications in many areas including fuzzy systems, fuzzy theory and its applications, soft computing systems, intelligent information processing, machine learning, knowledge management, pattern recognition, and neural networks. Traditional AI technologies and applications have challenges in processing massive data, large-scale communication, as well as collaborative computing of various algorithms, which thus require heavy use of high-performance computers and accelerators. To meet these challenges, parallel and distributed computing has been introduced. The objective of this special issue is to bring together parallel and distributed computing and $\mathrm{AI}$ communities to present the fuzzy-related applications and solutions to performance issues, and also to present how AI can be used to solve HPC problems.

Totally nine papers were selected for publication in the IJFS special issue on advances in parallel and distributed

Kenli Li

lk1@hnu.edu.cn

Guoqing Xiao

guoqing.xiao@uwaterloo.ca

Maozhen Li

Maozhen.Li@brunel.ac.uk

Lipo Wang

ELPWang@ntu.edu.sg

1 University of Waterloo, Waterloo, Canada

2 Hunan University, Changsha, China

3 Brunel University, Uxbridge, UK

4 Nanyang Technological University, Singapore, Singapore computing for fuzzy systems according to the review criterion on completeness and technical contributions. The nine papers represent a selection of extended excellent contributions presented at the 14th International Conference on Natural Computation, Fuzzy Systems and Knowledge Discovery (ICNC-FSKD 2018), Huangshan, China, July 28-30, 2018, and organized by Hefei University of Technology and Hunan University. ICNC-FSKD is a premier international forum for scientists and researchers to present the state of the art of data mining and intelligent methods inspired from nature, particularly biological, linguistic, and physical systems, with applications to computers, circuits, systems, control, communications, and more.

This special issue includes the following papers: "A fuzzy support vector machine enhanced convolutional neural network for recognition of glass defects," "Analysis of the impact of battlefield environment on military operation effectiveness using fuzzy influence diagram," "Fuzzy interacting multiple model $\mathrm{H}$-infinity particle filter algorithm based on current statistical model," "Fuzzy model applied in risk perception and price forecasts-based on laboratory experiment data," "Multi-granulation rough sets and uncertainty measurement for multi-source fuzzy information system," "Pairwise constrained fuzzy clustering: relation, comparison and parallelization," "Payments per claim model of outstanding claims reserve based on fuzzy linear regression," "PCPD: a parallel crime pattern discovery system for large-scale spatio-temporal data based on fuzzy clustering," and "Probability/possibility systems for modeling of random/fuzzy information with parallelization consideration," which cover the topics of fuzzy theory, systems, and applications, machine learning, cloud computing, parallel and distributed processing, and other applications in high-performance computing for 
fuzzy theory. It is hoped that this IJFS issue will make a good reference material and be of great use for readers in computer science, system engineering, artificial intelligence, etc.

The research was partially funded by the National Key R\&D Program of China (Grant No. 2018YFB0203800). The four guest editors wish to thank
Professor Shun-Feng Su, the Editor-in-chief in the International Journal of Fuzzy Systems, for providing the opportunity to edit this special issue and for his guidance throughout the edition process. We would also like to thank the referees who have critically evaluated the papers within the short time. Finally, we hope the readers will share our joy and find this special issue very useful. 